\section{ABSTRACT}

Graft versus host disease (GVHD) occurs after a hematopoietic stem cell transplantation (HSCD) when the donor's immune system rejects the recipient's body, leading to significant morbidity and mortality. Increased numbers of chronic GVHD (c-GVHD) patients are likely to be seen by dental professionals because of the advances made in transplantation. The oral cavity may be the primary or the only site of c-GVHD and may have persistent lesions after resolution has occurred in other areas.

Approximately $80 \%$ of patients with extensive c-GVHD present some type of oral involvement, including xerostomia, oral pain and lesions. Dental and oral care can be challenging for these patients. This paper discusses the manifestations and treatment of oral c-GVHD and presents the case history of a 15month-old girl who developed severe oral GVHD with an unusual periodontal presentation and early loss of primary teeth.

\section{KGY WORDSE oral GVHD}

hematopoietic stem cell transplant, children, oral manifestations

\title{
Severe gingival recession and early loss of teeth in a child with chronic graft versus host disease: a case report
}

\author{
Marcio A. da Fonseca, DDS, MS;" Carol Anne Murdoch-Kinch, DDS, PhD² \\ ${ }^{1}$ Clinical Associate Professor, Section of Pediatric Dentistry, The Ohio State University College of \\ Dentistry/Columbus Children's Hospital, Columbus, Ohio; ${ }^{2}$ Associate Professor, Department of \\ Hospital Dentistry, University of Michigan School of Dentistry, Ann Arbor, Michigan. ${ }^{*}$ Corresponding \\ author e-mail: dafonsem@chi.osu.edu
}

Spec Care Dentist 27(2): 59-63, 2007

\section{Introduction}

The success of hematopoietic stem cell transplantation (HSCT) in children can be related to improved HSCT strategies, better supportive care, and a greater understanding of the transplant process, immune status, tolerance, and graft versus host disease (GVHD).' GVHD occurs when the donor's immune system rejects the recipient's body, and has been associated with significant morbidity and mortality.' The autoreactive Tcells mediate damage through direct tissue attack and secretion of cytokines but the disease's basic pathophysiology is still poorly defined ${ }^{2-5}$ Careful human leukocyte antigen matching is extremely important for a successful outcome. GVHD can be prevented through the administration of methotrexate, cyclosporine, prednisone, and/or tacrolimus and depletion of donor T-cells. 1.60 The disease can be divided into acute (occurring within 80 days after the transplant) and chronic stages (starting after 80 days). ${ }^{2,5}$ Chronic GVHD (c-GVHD) can be classified as progressive (evolving directly from the acute form), quiescent or interrupted (following a period of resolution of acute GVHD) or de novo (developing with no prior history of GVHD). The chronic form represents the majority of the cases and has the best prognosis of all types. ${ }^{2,3}$ The most common staging system designates GVHD as limited when only localized skin involvement or hepatic dysfunction is present, or extensive when it involves the skin, liver, eyes, minor salivary glands, oral mucosa, or any other target organ..$^{24}$ Children receiving unrelated donor grafts have a c-GVHD incidence rate of approximately $40 \%$; however, the true incidence is difficult to quantify due to the marked heterogeneity in GVHD prophylaxis and stem cell sources. ${ }^{2,6}$

Increased numbers of c-GVHD survivors are likely to be seen in dental practice because of higher rates of survival due to advances in HSCT. Painful oral lesions may significantly impede the ability of persons with c-GVHD to eat, swallow, and carry out personal oral hygiene. Dental and oral care can be challenging for these patients. This paper discusses the manifestations and treatment of oral c-GVHD and reports on a 15-month-old girl with GVHD who had an unusual periodontal presentation, which resulted in early loss of her primary teeth.

\section{Case report}

The female patient was diagnosed with acute non-lymphocytic leukemia, type Mo (minimally differentiated), at the age of 7 months. She was treated according to protocol 2891 of the Children's Cancer Group, initially receiving two cycles of 
dexamethasone, cytarabine, thioguanine, etoposide and rubidomycin. Her medical history showed that she had $7 q$ deletion, high initial white cell count

$\left(>100,000 / \mathrm{mm}^{3}\right)$ and failure to respond to the induction therapy which made her leukemia high risk; therefore HSCT was recommended. The patient had a pretransplant oral screening in our dental clinic which revealed no abnormalities. The parents were advised about oral care and the possible acute and long-term effects of treatment in the craniofacial complex. She received a full-match, unrelated donor HSCT at the age of 15 months. Her conditioning regimen consisted of busulfan, cytarabine and cyclophosphamide (Cytoxan), and GVHD prophylaxis was done with tacrolimus and methotrexate. She was discharged on day +21 on several medications, including antibiotics, antiviral and antifungal agents. On day +45 , she returned with acute GVHD in the skin which was controlled with oral tacrolimus. The day +100 work-up showed that the disease had progressed to chronic, involving the skin and the intestines. On day +236 , a diagnosis of recurrent leukemia was made and she subsequently received donor lymphocyte infusions. On day +622 , an oral examination revealed atrophic lips, decreased oral opening and an ulcerated lesion on the dorsum of the tongue. A dexamethasone rinse $(1 \mathrm{mg} / \mathrm{ml})$ was prescribed with orders to swab the oral cavity for approximately two minutes four to six times a day for seven days. This treatment led to the resolution of the lesion. One month later, new tongue ulcers, complete ankyloglossia, and gingival recession with exposure of the cervical third of the incisor roots were seen. Some of the incisors had a mobility of grade 2 . Severe oral dryness was seen based on the patient's subjective complaint of dryness and clinical assessment of mucosal dryness and absence of saliva pooling on the floor of the mouth. Because she was very sick, it was decided not to treat the teeth at that point. Cultures were difficult to obtain due to her age-appropriate non-compliant behavior and excessive oral dryness. A dexamethasone swab was used but it

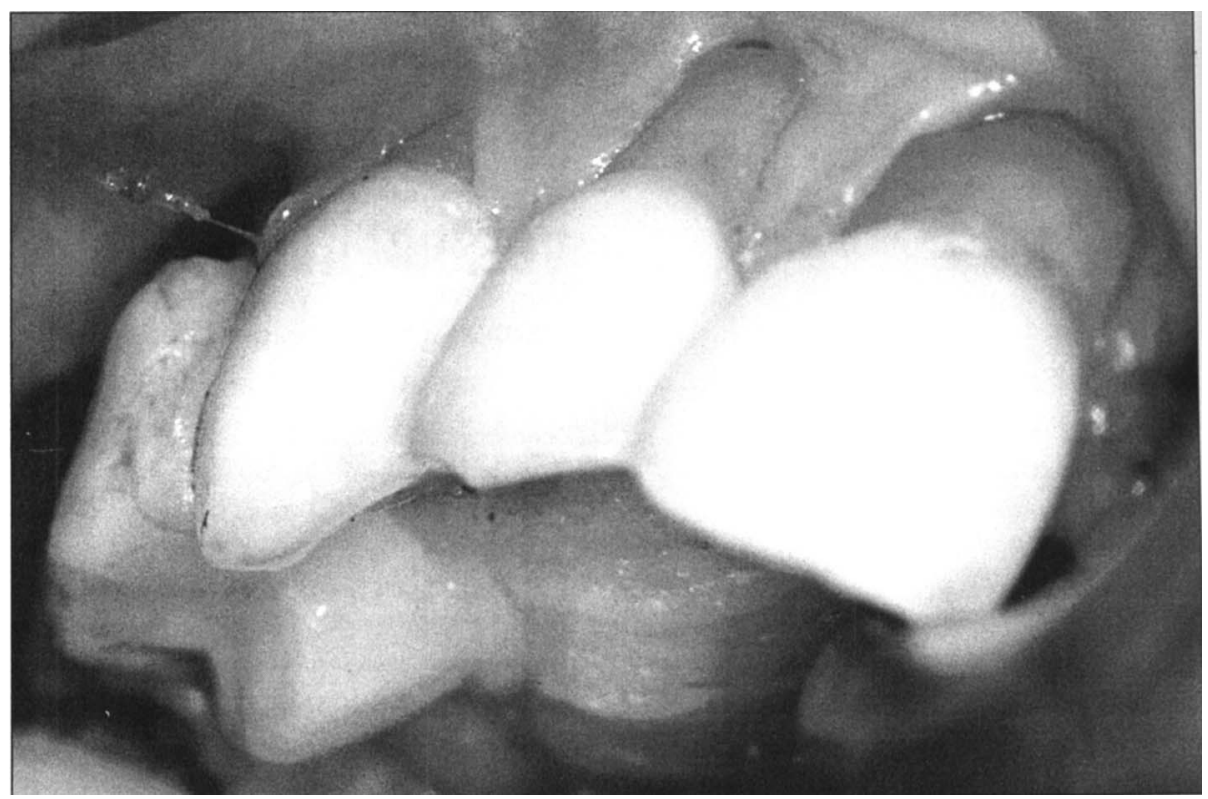

Figure 1. Severe gingival recession of the maxillary teeth.

was not effective, thus fluocinonide $0.05 \%$ gel was prescribed. It was applied with a cotton tip to the affected areas twice daily for 10 days. Her clinical signs and symptoms improved significantly with this treatment. A small amount of fluoride gel on the toothbrush was prescribed to be used daily at bedtime for caries prevention. Four months later, the patient returned with complaints of oral pain. She now had a very limited oral opening due to perioral fibrosis. She also had facial edema, oral dryness, extensive lichenoid changes in the buccal mucosa, complete ankyloglossia, tongue ulcerations, and generalized gingival recession exposing the roots of most primary teeth (Figures 1 and 2) with increased mobility of most incisors. An application of $2 \%$ viscous lidocaine with a cotton tip on the lesions was suggested for pain relief. She was prescribed clobetasol $0.05 \%$ gel to be applied to the oral mucosa twice daily for 10 days. A few days later, the patient needed a medical procedure under general anesthesia and while she was orally intubated, occlusal restorations were placed on teeth \#B and \#S and teeth \#E, $F, G, O$, and $P$ were extracted due to their mobility and poor prognosis.

Radiographs were made but were not of diagnostic quality due to the difficulties encountered in placing the films in her small oral cavity along with the other problems such as the oral intubation, ankyloglossia, fibrosis, etc. No soft tissue biopsy was taken because both the medical and dental teams agreed that the clinical picture was consistent with oral c-GVHD and that elective invasive procedures should be avoided because of her immunosuppression. She was given intravenous clindamycin for endocarditis prophylaxis because she had a central line placed and she was allergic to cephalosporins. ${ }^{i}$ Oral cultures grew gram-negative cocci and rods as well as coagulase-positive staphylococcus. No viruses were identified in the cultures. Later that month, she developed extensive bilateral erosive lesions on the buccal mucosa with severe oral pain which was controlled with oxycodone. A relapse of her leukemia was diagnosed and she died after a complicated hospital course a few days later.

\section{Discussion}

Chronic GVHD is the most common non-relapse problem affecting survivors of allogeneic HSCT, and is associated with pronounced immunosuppression. Therefore, patient/family education, prophylaxis for infection and rigorous medical and dental follow-up are impor- 


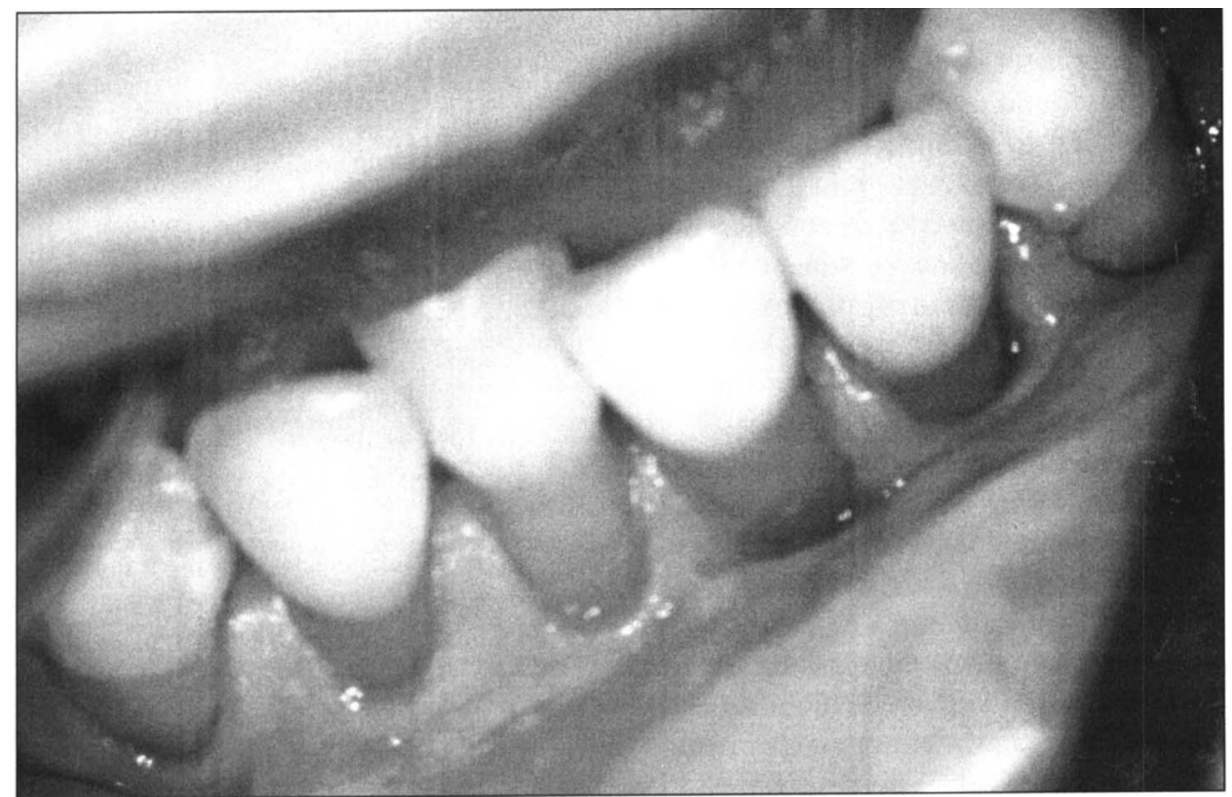

Figure 2. Severe gingival recession of the mandibular teeth.

tant measures for early diagnosis and management of the disease and opportunistic infections..$^{2.3}$ Biopsies and other tests should only be done to rule out alternative etiologies and to confirm the diagnosis of GVHD. ${ }^{2,3}$ Systemic treatment for GVHD includes prednisone, cyclosporine, tacrolimus, azathioprine, thalidomide, clofazimine, psoralen plus ultraviolet A (PUVA), and extracorporeal photochemotherapy (ECP). Treatment needs to continue for at least six to nine months or until all clinical and pathological evidence of the disease has disappeared. ${ }^{1-3,+, 8}$ Deaths from C-GVHD are usually due to infection. ${ }^{3,4}$ In pediatric patients, the disease and/or its treatment can inhibit growth, although most children catch up once the disease is controlled and immunosuppression. has occurred. ${ }^{2.3}$

A review of the literature showed only a few studies of oral c-GVHD manifestations in children, all of which stressed the need for systematic research of the disease in pediatric patients and development of adequate treatment strategies. ${ }^{9.10 .11}$ The oral cavity may be the primary or only site of c-GVHD problems and may show persistent lesions after its resolution in other areas. ${ }^{4.5}$ Approximately $80 \%$ of patients with extensive c-GVHD present some type of oral problems. ${ }^{4}$ Increasing oral dryness, pain and/or lesions around day +100 can be an indication of the disease." The most comprehensive pediatric study was done by Treister et al, ${ }^{4}$ who described the oral findings in 49 consecutive patients seen in a pediatric multidisciplinary cGVHD clinic. Almost half of the children had a diagnosis of oral c-GVHD. The most common findings were reticular, erythematous and ulcerative lesions, atrophic glossitis, gross caries and soft tissue fibrosis, which caused limited opening of the mouth due to either sclerosis of the facial skin or limited mobility of the soft tissues. Mucoceles and some soft tissue growths were also noted. A few patients complained of oral pain and $20 \%$ avoided certain foods, particularly spicy and acidic products, although none had difficulties swallowing. There was an apparent lack of salivary gland involvement with only a few subjects reporting dry mouth (xerostomia) or objective evidence of hyposalivation. Dahllof et al. ${ }^{10}$ described six children with c-GVHD who presented with erythema of the mucous membranes, tongue atrophy and lichenoid changes in the buccal mucosa

Clinicians rely on oral biopsies and oral examinations to diagnose the disease and both have high predictive values of extensive c-GVHD. ${ }^{45.12}$ Evaluation of biopsy of the lower labial minor salivary glands show ductal necrosis, sialadenitis, epithelial lymphocytic infiltration and acinar destruction. ${ }^{22}$ Oral c-GVHD closely resembles autoimmune connective tissue diseases such as lichen planus (oral mucosa) and Sjögren's syndrome (salivary glands), with mucosal erythema, atrophy, pseudomembrane ulcerations, hyperkeratotic striae, including plaques and papules. ${ }^{1-13}$ Reticular lesions rarely require treatment whereas erythematous and ulcerative lesions need aggressive intervention. ${ }^{9}$ In the patient described in this paper, ankyloglossia and perioral sclerodermatous changes increase the difficulties with nutrition and oral hygiene as well as dental care.

Patients with HSCT and c-GVHD may be at increased risk for periodontal disease because of immunosuppression and alterations in the subgingival flora. The most striking aspect of this case report was the severe, progressive, generalized gingival recession with exposure of the roots of primary teeth and bone loss, which led to premature tooth loss. We were unable to find any previous reports of pediatric GVHD that presented such extensive periodontal involvement. No systematic studies of periodontal health in c-GVHD have been published. Pattni et al.$^{14}$ observed a slight loss of attachment in adult HSCT recipients but did not follow them long enough to describe any effects of GVHD on the periodontium.

C-GVHD can injure salivary glands, leading to xerostomia and the formation of mucoceles. ${ }^{4.11,13}$ There is a direct correlation between the degree of hyposalivation and GVHD severity, and assessing salivary gland function is a simple, non-invasive way to monitor the disease. ${ }^{15}$ The saliva shows alteration in its biochemical and immunological composition, making the patient more susceptible to epithelial insults and fulminate infections." Surprisingly, rampant caries doesn't develop often despite the marked xerostomia.' Optimal oral hygiene is important to reduce local infection. Topical fluoride should be used for caries prevention. ${ }^{4.5}$ A review of current therapies for xerostomia associated 
with cancer therapies is described elsewhere. $^{17}$

The best strategy for management of oral $c-G V H D$ relies on successful systemic treatment but in many patients that may not completely resolve severe atrophic and ulcerative lesions. ${ }^{+5}$

Extracorporeal photochemotherapy, for example, does not prevent evolution of the oral lesions. ${ }^{.}$In such situations, management is focused on the treatment of specific lesions, pain control, relief of dryness and maintenance of oral health.' Fluocinonide and clobetasol steroid gels can be used for localized lesions; dexamethasone or betamethasone rinses may be used for generalized involvement. ${ }^{4.5 .16}$ As seen in our patient, their efficacy had mixed results, partly because their use is restricted due to difficulty retaining the topical medication locally and allowing uptake by the lesional tissue. ${ }^{4.5}$ Rinses with cyclosporine, budesonide and azathioprine, tacrolimus ointment, PUVA therapy, and ultraviolet $B$ irradiation have also been used. ${ }^{16.18-22}$ When one or two large lesions are present, intralesional injections with triamcinolone or triamcinolone acetonide may be beneficial.' Thalidomide may be considered for patients who don't respond to systemic and/or topical steroids. ${ }^{5}$ Resistant symptomatic oral lesions may be caused by overgrowth or reactivation of herpes virus or cytomegalovirus. ${ }^{4}$ Careful follow-up, with the use of appropriate cultures and even biopsy with immunostaining for herpes group viruses, can ensure timely treatment of these infections which usually have an atypical presentation. $^{5}$

Topical anesthetics, systemic analgesia and soothing saline rinses help control oral pain.+ Anesthetic solutions, such as $2 \%$ viscous lidocaine, must be used judiciously. Patients should avoid gargling or swallowing local anesthetic solutions to keep the gag reflex intact. Moreover, the frequent use (more than once every 30 to 60 minutes) of anesthetic solutions may give rise to toxic blood levels with complications, especially where injured mucosal tissues are present. ${ }^{23}$ Systemic pain medications should start with non-narcotics and progress to agents containing codeine or hydrocodone or stronger opiates such as meperidine and morphine. ${ }^{5}$ During the patient's last hospitalization, intravenous morphine was not enough to control oral pain; only hydromorphone could accomplish this. Throughout treatment, she also used oral methadone and oxycodone when sublingual morphine was ineffective.

The risk of secondary cancers is highest among children who undergo a HSCT procedure before 10 years of age. ${ }^{24}$ Male gender and c-GVHD are significantly linked with a higher risk of squamous cell carcinoma of the mouth. ${ }^{23}$ Therefore, any suspicious lesions seen in long-term survivors of HSCT must be evaluated promptly. Non-gingival, benign soft tissue growths - possibly linked to cyclosporine or tacrolimus use - have also been reported in patients with GVHD, 3,9

\section{Conclusion}

Very little research has been done to assess oral c-GVHD problems in children. The disease's management in young patients such as ours is a challenge because of issues of compliance and the fact that most medications are available for adult use only. With the increasing numbers of long-term HSCT survivors, dental professionals will be faced with treating the oral problems of this population. In order to ensure survival and a better quality of life for the child, close clinical and research collaboration between the medical and dental teams, together with education of general dentists and specialists, is critical for early diagnosis and effective treatment of the disease.

\section{References}

1. Gross $\mathrm{TG}$, Egeler RM, Smith FO. Pediatric hematopoietic stem cell transplantation. Hematol Oncol Clin North Am 2001;15:795808.

2. Higman MA, Vogelsang B. Chronic graft versus host disease. Br J Haematol 2004:125:435-54.

3. Lee SJ, Vogelsang G, Flowers ME. Chronic graft-versus-host disease. Biol Blood Marrow Transplant 2003;9:215-33.

4. Schubert MM, Sullivan KM. Recognition, incidence, and management of oral graftversus-host disease. NCI Monogr 1990;9:135-43.

5. Woo SB, Lee SJ, Schubert MM. Graft-vs.host disease. Crit Rev Oral Biol Med 1997;8:201-16.

6. Sanders JE. Bone marrow transplantation for pediatric malignancies. Pediatr Clin North Am 1997;44:1005-20.

7. Dajani AS, Taubert KA, Wilson W, et al. Prevention of bacterial endocarditis. Recommendations by the American Heart Association. JAMA 1997;277;1794-801.

8. Kanold J, Paillard C, Haile P, D'Incan $M$, Bordigoni P, Demeocq F. Extracorporeal photochemotherapy for graft versus host disease in pediatric patients. Transfus Apher Sci 2003;28:71-80.

9. Treister NS, Woo SB, O'Holleran EW, Lehmann LE, Parsons SK, Guinan EC. Oral chronic graft-versus-host disease in pediatric patients after hematopoietic stem cell transplantation. Biol Blood Marrow Transplant 2005;11:721-31.

10. Dahllof G, Heimdahl A, Modeer T, Twetman $S$, Bolme $\mathrm{P}$, Ringden O. Oral mucous membrane lesions in children treated with bone marrow transplantation. Scand J Dent Res 1989:97:268-77.

11. Nicolatou-Galitis O, Kitra V, Van VlietConstantinidou $\mathrm{C}$, et al. The oral manifestations of chronic graft-versus-host disease (cGVHD) in paediatric allogeneic bone marrow transplant recipients. J Oral Pathol Medic 2001;30:148-53

12. Schubert MM, Sullivan KM, Morton TH, et al. Oral manifestations of graft-v-host disease. Arch Intern Med 1984;144:1591-5.

13. Nakamura $S$, Hiroki $A$, Shinohara $M$, et al. Oral involvement in chronic graft-versushost disease after allogeneic bone marrow transplantation. Oral Surg Oral Med Oral Pathol Oral Radiol Endod 1996;82:556-63.

14. Pattni R, Walsh LJ, Marshall RI, Cullinan MP, Seymour GJ, Bartold PM. Changes in periodontal status of patients undergoing bone marrow transplantation. J Periodontol 2000;71:394-402.

15. Nagler RM, Nagler A. Sialometrical and sialochemical analysis of patients with chronic graft-versus-host disease - a prolonged study. Canc Invest 2003;21:34-40. 
16. Epstein JB, Nantel S, Sheoltch SM. Topical azathioprine in the combined treatment of chronic oral graft-versus-host disease. Bone Marrow Transplant 2000;25:683-7.

17. Nieuw Amerongen AV, Veerman EC. Current therapies for xerostomia and salivary gland hypofunction associated with cancer therapies. Support Care Cancer 2003;11:226-31

18. Epstein JB, Reece DE. Topical cyclosporine $A$ for treatment of oral chronic graft-versushost disease. Bone Marrow Transplant $1994: 13: 81-6$

19. Vogelsang GB, Wolff D, Altomonte $\mathrm{V}$, et al. Treatment of chronic graft-versus-host disease with ultraviolet irradiation and psoralen (PUVA). Bone Marrow Transplant 1996;17:1061-7.

20. Elad S, Garfunkel AA, Enk CD, Galili D, Or R. Ultraviolet B irradiation: a new therapeutic concept for the management of oral manifestations of graft-versus-host disease. Oral Surg Oral Med Oral Pathol Oral Radiol Endod 1999;88:444-50.

21. Elad S, Or R, Garfunkel AA, Shapira MY. Budesonide: a novel treatment for oral chronic graft versus host disease. Oral Surg Oral Med Oral Pathol Oral Radiol Endod 2003;95:308- i 1
22. Eckardt A, Starke O, Stadler M, Reuter C, Hertenstein B. Severe oral chronic graftversus-host disease following allogeneic bone marrow transplantation: highly effective treatment with topical tacrolimus. Oral Oncol 2004:40:811-4

23. Sharon $\mathrm{E}$, Cohen $\mathrm{G}$, Zylbert-Katz $\mathrm{E}$, et al. Systemic absorption of lidocaine after topical application for the treatment of oral mucositis in bone marrow transplantation patients. J Oral Pathol Med 1999;28:170-2.

24. Curtis RE, Rowlings PA, Deeg HJ, et al. Solid cancers after bone marrow transplantation. N Engl J Med 1997;336:897-904. 